\title{
Approximation Algorithms for the Capacitated Multi-Item Lot-Sizing Problem via Flow-Cover Inequalities
}

\author{
Retsef Levi \\ Sloan School of Management, MIT, Cambridge, MA, 02139, USA \\ email: retsef@mit.edu \\ Andrea Lodi \\ University of Bologna, Viale Risorgimento 2, 40136 Bologna, Italy. \\ email: andrea.lodi@unibo.it \\ Maxim Sviridenko \\ IBM T.J. Watson Research Center, P.O. Box 218, Yorktown Heights, NY 10598. \\ email: sviri@us.ibm.com
}

\begin{abstract}
We study the classical capacitated multi-item lot-sizing problem with hard capacities. There are $N$ items, each of which has specified sequence of demands over a finite planning horizon of $T$ discrete periods; the demands are known in advance but can vary from period to period. All demands must be satisfied on time. Each order incurs a time-dependent fixed ordering cost regardless of the combination of items or the number of units ordered, but the total number of units ordered cannot exceed a given capacity $C$. On the other hand, carrying inventory from period to period incurs holding costs. The goal is to find a feasible solution with minimum overall ordering and holding costs.

We show that the problem is strongly NP-hard, and then propose a novel facility location type LP relaxation that is based on an exponentially large subset of the well-known flow-cover inequalities; the proposed LP can be solved to optimality in polynomial time via an efficient separation procedure for this subset of inequalities. Moreover, the optimal solution of the LP can be rounded to a feasible integer solution with cost that is at most twice the optimal cost; this provides a 2-approximation algorithm which is the first constant approximation algorithm for the problem. We also describe an interesting on-the-fly variant of the algorithm that does not require solving the LP a-priori with all the flow-cover inequalities. As a by-product we obtain the first theoretical proof regarding the strength of flow-cover inequalities in capacitated inventory models.
\end{abstract}

Key words: Inventory management; Approximation ; Flow-cover inequalities ; Algorithms

MSC2000 Subject Classification: Primary: 90B05; Secondary: 68W25,

OR/MS subject classification: Primary: inventory/production , approximations/heuristics; Secondary: production/scheduling, approximations/heuristics

1. Introduction The issue of capacity constraints arises in many practical and theoretical inventory management problems as well as in problems in other application domains, such as facility location problems. In most practical inventory systems there exist capacity constraints that limit the quantities that one can order, ship or produce. Unfortunately, it is often the case that models with capacity constraints are computationally far more challenging than their counterpart models with no capacity constraints. In particular, in many problems with capacity constraints computing optimal policies and sometimes even feasible policies is a very challenging task.

In recent years there has been an immense amount of work to develop integer programming methods for solving hard, large-scale deterministic inventory management problems. (We refer the reader to the recent book of Pochet and Wolsey [20].) A major part of this work has been focused on constructing strong formulations for the corresponding inventory models. In fact, it is essential to have an integer programming formulation with a strong linear programming relaxation. Stronger formulations are achieved by identifying valid inequalities that are satisfied by all feasible integral solutions and cut off fractional solutions. Another key aspect within an integer programming framework is the ability to construct good feasible integer solutions to the corresponding model. This has been known to have a huge impact on decreasing the computational effort involved. In models with capacity constraints, finding good feasible solutions can be very challenging.

In this paper, we study the classical capacitated multi-item lot-sizing problem, which is an extension of the single-item economic lot-sizing problem [20]. Next, we propose a novel facility location type linear program (LP), and show how to round its optimal solution to a feasible integral solution with cost that is guaranteed to be at most twice the optimal cost. This is called a 2-approximation algorithm, that is, 
the cost of the solution constructed by the algorithm is guaranteed to be at most twice the optimal cost. This is the first constant approximation algorithm for this problem. The LP relaxation is based on a variant of a well-known class of valid inequalities called flow-cover inequalities. These inequalities have been introduced over two decades ago [18] and have been shown empirically to be very effective in solving several inventory and facility location problems with capacity constraints [1, 20]. (In Section 3 below, we discuss the relevant literature on flow-cover inequalities in more detail.) Our results have several significant contributions: (i) To the best of our knowledge, this is the first theoretical evidence for the strength of flow-cover inequalities applied to capacitated inventory models. All the previous theoretical results have been obtained for fixed-charge single-node problems (see Section 3 below for details.); (ii) Our approach provides a conceptually simple way to generate provably good feasible solutions, and can be easily implemented within an integer programming framework; (iii) Several of the newly proposed algorithmic ideas in this paper have a promising potential of applicability in other inventory models with capacity constraints. Moreover, we believe that they can be used to develop strong LP relaxations and LP-based approximation algorithms for the capacitated facility location problem.

The model. The details of the inventory model discussed in this paper are as follows. There are $N$ items indexed by $i=1, \ldots, N$, each of which has a specified sequence of demands over a finite planning horizon of $T$ discrete periods indexed by $t=1, \ldots, T$. The demand of item $i$ in period $t$ is denoted by $d_{i t}$. The demands are known in advance but can vary from period to period. Moreover, all of the demands must be fully satisfied on time, that is, $d_{i t}$ must be fully ordered by time period $t$. At the beginning of each period $s=1, \ldots, T$, it is possible to place an order for any subsets of items, and this incurs a fixed ordering cost $K_{s}$ regardless of the subsets of items or the (possibly fractional) number of units ordered from each item. However, the overall quantity of units ordered in period $s$ cannot exceed a certain capacity limit $C_{s} \geq 0$. These are usually called hard capacity constraints in contrast to soft capacity constraints, where in each period $s$, the order is placed in batches, each of which has capacity $C_{s}$ and incurs an additional fixed ordering cost $K_{s}$. We consider the special case with uniform capacities, i.e., $C_{s}=C$, for each $s=1, \ldots, T$.

The units ordered in period $s$ are assumed to arrive instantaneously, and can be used to satisfy demands in that period and subsequent periods. The fixed ordering cost is balanced with a cost to maintain physical inventory that is called the holding cost. In most of the existing literature the holding costs are linear and additive. Specifically, for each item $i$ and period $t$, there is a holding cost parameter $h_{i t} \geq 0$ that denotes the per-unit cost to carry one unit of item $i$ in inventory from period $t$ to period $t+1$. Following Levi, Roundy and Shmoys [14], we model the holding cost in a more general way. For each demand point $(i, t)$ and a potential order $s \leq t$, let $h_{s t}^{i} \geq 0$ be the per-unit cost of holding one unit of item $i$ in inventory from period $s$ to period $t$. The only assumption is that, for a fixed $(i, t)$, the parameters $h_{s t}^{i}$ are non-increasing in $s$. (This implies that if $d_{i t}$ is ordered from a closer period to $t$ the resulting holding cost is not bigger.) The way we model the holding cost is more general, and can capture several important phenomena such as perishable goods. We also note that we can incorporate a per-unit ordering cost into the holding cost parameters. The goal is to find a feasible policy that satisfies all of the demands on time and has minimum overall ordering and holding cost.

Literature review. As we already mentioned, this is a classical model in inventory theory that has been studied by several researchers throughout the years. The special case with a single item $(N=1)$ and uniform capacities is polynomially solvable both with hard capacities [12] and soft capacities [19]. This is usually called single-item capacitated economic lot-sizing problem. Moreover, there are known extended $L P s$, that is, LPs with integrality property that provide an exact description of the set of feasible solutions. The single item problem with non-uniform capacities is known to be weakly NP-hard [12], but there is a fully polynomial time approximation scheme (FPTAS) [21]. For results on other variants of single-item models, we refer the reader to $[6,20]$.

Federgruen, Meisner and Tzur [11] have studied the model discussed in this paper with traditional (i.e., linear and additive) holding costs, but with additional fixed item ordering costs that are incurred in each period, in which item $i$ is ordered. Under the assumption that all of the demands and the cost parameters are uniformly bounded by constants, they have proposed a dynamic-programming-based algorithm, and shown that it is asymptotically optimal as the number of periods increases to infinity. In a subsequent paper [10], they provide a probabilistic analysis of the algorithm. Another dynamic-programming-based algorithm for a special case of the model discussed in this paper has been proposed by Anily and Tzur [3]. 
They have studied a model with traditional holding costs and stationary cost parameters, i.e., $h_{i t}=h$ and $K_{t}=K$, for each $i$ and $t$. However, the running time of their algorithm grows exponentially fast in the number of items, and thus, it is not practical unless there are few items. In a recent paper Anily, Tzur and Wolsey [4] have considered the same model with time-dependent cost parameters, but with the additional monotonicity assumption on the holding costs. In particular, the assumption is that the items are indexed, such that each item has higher holding costs than all items of smaller index, uniformly for all periods. Specifically, $h_{1 t} \leq h_{2 t} \leq \cdots \leq h_{N t}$, for all periods $t=1, \ldots, T$. For this problem, they have proposed an extended linear programming formulation with $O\left(N \times T^{2}\right)$ constraints and variables that solves the problem to optimality. This implies that this special case is polynomially solvable. In another recent result, Even at al. [9] have considered a model with multiple items, unit demands, nonuniform capacities and holding cost structure that is a special case of the one considered by Anily, Tzur and Wolsey [4]. Specifically, the holding cost of each demand point is zero until some point when it jumps to infinity. Thus, demand points can be viewed as intervals that one needs to stab by opening capacitated stabbing points, where opening a stabbing point incurs a fixed cost. Even et al. [9] have given a polynomial time optimization algorithm for this model based on dynamic programming. They then extend their approach to capture holding cost structure that is similar to the one considered by Anily, Tzur and Wolsey. We note that the assumption of unit demands is crucial as otherwise the problem is known to be NP-hard $[12]$.

Our results and techniques. Our first result shows that the capacitated multi-item lot-sizing problem with hard or soft capacities is strongly NP-hard. This implies that the monotonicity assumption of Anily, Tzur and Wolsey [4] is somewhat essential to get a polynomial time optimization algorithm.

Next, we propose a novel facility location type LP relaxation for the problem that is different than the one used by Anily, Tzur and Wolsey [4]. Our LP is based on the family of flow-cover inequalities in the same spirit as the LP proposed by Aardal, Pochet and Wolsey for the capacitated facility location problem [2]. However, it incorporates only a subset of the class of flow-cover inequalities: there are exponentially many inequalities in this subset, but we show that they can be separated in polynomial time. Thus, the LP can be solved optimally in polynomial time, using the Ellipsoid method. We then use an extremely simple rounding algorithm. The optimal solution of the LP relaxation is scaled by a suitably chosen factor, and the scaled solution is used to execute a randomized rounding procedure that outputs the sequence of periods in which orders are placed. Given the output of the first phase, demands are assigned to orders by solving the induced transportation problem, and this minimizes the resulting holding costs. The main challenge in the worst-case analysis is to show that the first phase of the algorithm opens enough capacity to serve all of the demands, and that the resulting solution is of low cost. This is done by exploiting the structure of the flow-cover inequalities. In particular, we show that together with the scaling in the first phase of the algorithm, they guarantee that the resulting transportation problem has a low cost feasible solution. This provides a randomized 2-approximation algorithm. The randomized procedure can be derandomized to provide a deterministic 2-approximation algorithm. As a by-product, we obtain the first theoretical proof of the strength of flow-cover inequalities in capacitated inventory models. As already mentioned, all previous results are restricted to fixed-charge single-node problems, see Section 3 below.

Finally, the insights from the worst-case analysis are used to construct an on-the-fly variant of the algorithm. Instead of solving the LP a-priori with all the corresponding flow-cover inequalities, we propose an iterative procedure. In each iteration, a well designed rounding procedure is applied to the optimal fractional solution of the LP relaxation. If this procedure comes to an end successfully, it can be shown that the resulting integral solution is feasible and has cost that is at most twice the optimal cost. On the other hand, if the procedure is terminated in the middle, it is guaranteed to identify a violated flow-cover inequality. The corresponding inequality is added to the LP, which is then solved again. The on-the-fly algorithm can be viewed as running the Ellipsoid method until termination or until the first time the rounding procedure is 'stuck', whereas then we are guaranteed to have a good feasible integral solution. We believe that the on-the-fly algorithm might be computationally more efficient, since it does not require solving the LP a-priori with all the flow-cover inequalities. This algorithmic approach is similar in spirit to what is discussed in Carr at al. [8] in the context of a single-node fixed charge problem.

The rest of the paper is organized as follows. In Section 2 we discuss the complexity of the multi-item capacitated lot-sizing problem. In Section 3, we describe the LP relaxation and discuss the flow-cover 
inequalities. In Section 4, we describe the rounding algorithms and the worst-case analysis.

\section{Complexity}

THEOREM 2.1 The capacitated multi-item lot-sizing problem with hard capacity constraints is strongly NP-hard even in the special case of unit demand, i.e. $d_{i t}=1$ for all items $i$ and time periods $t$.

Proof. We define a reduction from the classical 3-PARTITION problem, in which we are given a set of $3 m$ jobs with sizes $p_{1}, \ldots, p_{3 m}$ such that $B / 4<p_{i}<B / 2$ for each $i=1, \ldots, 3 m$ and some integer $B$. The goal is to partition the set of jobs into $m$ groups $S_{1}, \ldots, S_{m}$, such that, for each $j=1, \ldots, m$, $\left|S_{j}\right|=3$ and $\sum_{i \in S_{j}} p_{i}=B$. It is well-known that this problem is strongly NP-hard [13].

Given an instance of the 3-PARTITION problem we define the following instance of the capacitated multi-item lot-sizing problem with hard capacities. Each job in the 3-PARTITION problem will correspond to an item in the inventory problem with exactly one unit demand point. All demands will arrive in the period $T$, that is, at the end of the planning horizon.

Let $\mathcal{S}$ be the set of all subsets $S$ of jobs, such that $|S|=3$ and $\sum_{i \in S} p_{i}=B$. That is, $\mathcal{S}$ contains all the sets $S$ which are feasible for the 3-PARTITION problem. We arbitrarily number the elements in $\mathcal{S}$ by $t=1, \ldots,|\mathcal{S}|$. Let $T=|\mathcal{S}|+1$ be the number of time periods and $C=3$ be the capacity of each order in the inventory instance. Each time period $t=1, \ldots, T-1$ in the inventory instance, corresponds to the respective set in $\mathcal{S}$ with the same index. That is, set $t \in \mathcal{S}$ corresponds to time period $t$.

Next we define the holding and ordering costs. Let $K_{t}=6 t$ for $t=1, \ldots, T-1$ and $K_{T}=6|T|^{2}$. In particular, we define the cost of ordering in the last period to be high enough to forbid such an order in any optimal solution. For each $i=1, \ldots, 3 m$ and $s=1, \ldots, T-1$, let $h_{s T}^{i}=2(T-s)+\lambda_{i s}$ where $\lambda_{i s}=0$ if the job $i$ belongs to the 3 -element set $s \in \mathcal{S}$ and $\lambda_{i s}=1$, otherwise. (Recall that period $s$ corresponds to the 3 -element set in $\mathcal{S}$ with the same index.) Observe that all the holding cost parameters are nonnegative, and that $h_{s T}^{i}<h_{s^{\prime} T}^{i}$, for each item $i$ and time periods $s>s^{\prime}$.

Consider any feasible solution to the 3-PARTITION problem. This solution consists of the sets $t_{1}, \ldots, t_{m} \in \mathcal{S}$. We consider the corresponding solution to the inventory problem. The orders are placed in the corresponding $m$ time periods $t_{1}, \ldots, t_{m}$. The demand $(i, T)$ is satisfied by the order placed in the time period $t_{k}$ that corresponds to the unique subset $t_{k} \in \mathcal{S}$, to which job $i$ belongs. The total cost of this solution is

$$
\sum_{k=1}^{m}\left(K_{t_{k}}+\sum_{i \in t_{k}} h_{t_{k}, T}^{i}\right)=\sum_{k=1}^{m}\left(6 t_{k}+6\left(T-t_{k}\right)\right)=6 m T .
$$

Consider now an optimal solution for the instance of the multi-item inventory problem with hard capacities defined above. We claim that this solution must have exactly $m$ orders. Assume otherwise, i.e., that there exists an optimal solution that has more than $m$ orders. Consider the earliest of these orders. Note that all the demands arrive in period $T$, and that $m$ orders would provide enough capacity to satisfy all demands. Therefore, we can cancel this order and satisfy all the demand points currently being served by this order from orders placed later in time. Since we canceled one order we decreased the ordering cost and because the monotonicity of the holding costs we also decreased the holding costs. This is a contradiction. Furthermore, since the above instance has only single unit demands, each demand point will be served from a single order.

Next we consider a feasible solution to the inventory model with exactly $m$ orders placed in periods $\tau_{1}, \ldots, \tau_{m}$. For each job $i=1, \ldots, 3 m$, let $s(i) \in\left\{\tau_{1}, \ldots, \tau_{m}\right\}$ be the period of the order, from which this demand point is served. By the definition of the cost parameters the total cost incurred by the above solution is $6 m T+\sum_{i=1}^{3 m} \lambda_{i, s(i)}$. This implies that, each feasible solution, has cost which is at least $6 m T$. Moreover, the value of $\sum_{i=1}^{3 m} \lambda_{i, s(i)}$ is equal to the number of demand points assigned to orders in periods that correspond to "wrong" sets of cardinality 3, i.e., sets that do not include that demand point. Thus, any optimal solution of the inventory model with value $6 m T$ corresponds to a feasible solution to the 3-PARTITION problem.

Therefore, solving the 3-PARTITION problem can be reduced to solving a special case of the capacitated multi-item lot-sizing problem with hard capacities. The theorem then follows. 
The next corollary implies that the variant of the capacitated multi-item lot-sizing problem with soft capacity constraints is also NP-hard.

COROLLARY 2.1 The capacitated multi-item lot-sizing problem with soft capacity constraints is strongly NP-hard even in the special case of unit demand, i.e. $d_{i t}=1$ for all items $i$ and time periods $t$.

Proof. The proof is identical to that of Theorem 2.1 above. Consider the instance of the inventory model that we have constructed but now with soft capacities. That is, we are allowed to order several batches in each period $t$, where each additional batch ordered has additional capacity of 3 units, and incurs additional ordering cost of $6 t$. Using similar arguments, one can show that each feasible solution to the 3PARTITION problem induces a solution to the inventory problem of cost $6 m T$, and each optimal solution to the inventory model has cost $6 m T$ only if it corresponds to a feasible solution to the 3-PARTITION problem.

The reduction in Theorem 2.1 above is originally written for a model with non-additive holding cost parameters $h_{s t}^{i}$ for each $(i, t)$ and $s \leq t$ that preserve nonnegativity and monotonicity in $s$. Next we show how to reduce any such problem to a modified capacitated multi-item lot-sizing problem with traditional additive holding cost parameters. This implies that the latter problem is also NP-hard. In the modified problem, we have an item $j$ for each demand point $(i, t)$ in the original problem (overall we have $N T$ items in the modified problem). For each item $j=(i, t)$, we define $d_{j t}=d_{i t}$ and $d_{j t^{\prime}}=0$, for each $t^{\prime} \neq t$. Also define $h_{j s}=0$, for each $s \geq t$ and $h_{j s}=h_{s t}^{i}-h_{s+1, t}^{i}$, for each $s<t$. Observe that because of the monotonicity of $h_{r t}^{i}$, it follows that $h_{j s} \geq 0$. The fixed ordering costs are kept the same. It can be verified that the modified problem is equivalent to the original problem. We have obtained the following theorem.

THEOREM 2.2 The capacitated multi-item lot-sizing problem with hard or soft capacity constraints and additive holding costs is strongly NP-hard even in the special case of unit demand, i.e. $d_{i t}=1$ for all items $i$ and time periods $t$.

3. A Flow-Cover-Inequality-Based LP Relaxation A natural Mixed Integer linear Programming (MIP) formulation of the capacitated multi-item lot-sizing problem can be obtained by using two sets of variables:

- For each $s=1, \ldots, T$, let $y_{s}$ be a binary variable that is equal to 1 if an order is placed in period $s$ and 0 otherwise.

- For each $i=1, \ldots, N, t=1, \ldots, T$ and $s=1, \ldots, t$, let $x_{s t}^{i}$ be the fraction of the demand $d_{i t}$ satisfied by an order placed in period $s$.

Moreover, for each $i=1, \ldots, N, t=1, \ldots, T$ and $s=1, \ldots, t$, let $H_{s t}^{i}=h_{s t}^{i} d_{i t}$ be the cost of holding the demand $d_{i t}$ if ordered in period $s$. The corresponding MIP formulation is as follows:

$\min \sum_{s=1}^{T} K_{s} y_{s}+\sum_{i=1}^{N} \sum_{s=1}^{T} \sum_{t=s}^{T} H_{s t}^{i} x_{s t}^{i}$

subject to

$$
\begin{aligned}
& \sum_{s \leq t} x_{s t}^{i}=1 \\
& x_{s t}^{i} \leq y_{s} \\
& \sum_{i=1}^{N} \sum_{t \geq s} d_{i t} x_{s t}^{i} \leq C y_{s} \\
& x_{s t}^{i} \geq 0 \\
& y_{s} \in\{0,1\} \\
& i=1,2, \ldots, N ; t=1,2, \ldots, T ; d_{i t}>0, \\
& i=1,2, \ldots, N ; s=1,2, \ldots, T ; t \geq s, \\
& s=1,2, \ldots, T, \\
& i=1,2, \ldots, N \text {, } \\
& s=1,2, \ldots, T \text {. }
\end{aligned}
$$

Constraints (2) guarantee that any positive demand is fully satisfied on time, while Constraints (3) state that an order cannot be used to satisfy a demand if it is not placed. Finally, Constraints (4) guarantee 
that the capacity $C$ of every order is not exceeded. In the objective function (1) the first part is associated with the ordering cost while the second measures the holding costs.

If we relax the integrality constraints to $0 \leq y_{s} \leq 1$, we get an LP relaxation that provides a lower bound on the cost of the optimal solution. However, this LP relaxation is weak in that the gap between its optimal value and the value of the optimal integral solution can be arbitrarily high. For example, consider an instance with a single item and 2 periods, no holding costs, fixed ordering costs $K_{1}=0$ and $K_{2}=1$, and demands $d_{1}=0$ and $d_{2}=C+1$. The optimal policy must open two orders incurring a cost of 1 . The optimal fractional solution can achieve a cost of $1 / C$ by setting $y_{1}=1, y_{2}=1 / C$, $x_{1,2}=C /(C+1)$ and $x_{2,2}=1 /(C+1)$. Thus, there is no hope of using this LP to construct constant factor approximation algorithms.

3.1 Flow-cover inequalities In this section, we introduce the class of flow-cover inequalities that we use to strengthen the LP induced by (1)-(6). Flow-cover inequalities were introduced by Padberg, Van Roy and Wolsey [18] over two decades ago in the context of the fixed charge single-node problem. In this problem there is a single-node of demand $D$ and a collection of $T$ capacitated arcs. The goal is to open arcs and send a flow of $D$ units to the demand node. Opening $\operatorname{arc} s$ incurs a fixed cost $K_{s}$, and sending flow over arc $s$ incurs a cost $h_{s}$ for each unit of flow. Padberg, Van Roy and Wolsey [18] have used flow-cover inequalities to construct an extended LP for this problem with uniform arc capacities. They have also shown that these flow-cover inequalities can be separated in polynomial time. Carr et al. [8] have shown that another variant of flow-cover inequalities can be used to construct an LP relaxation for the fixed-charge single-node problem with nonuniform capacities, whose optimal solution can be rounded to a feasible solution with cost that is at most twice the optimal cost. Carnes and Shmoys [7] have used the same LP to construct a primal-dual algorithm with the same worst-case performance guarantee. Aardal, Pochet and Wolsey [2] have used aggregation of constraints to apply the flow-cover inequalities to multi-location problems, specifically, hard capacitated facility location problems. They have reported that flow-cover inequalities seem to be effective in narrowing the integrality gap and enhance integer programming solution procedures. However, to the best of our knowledge there has been no theoretical analysis regarding the strength of flow-cover inequalities in facility location or inventory models with multiple items.

In the spirit of [2], we next introduce flow-cover inequalities for the capacitated multi-item lot-sizing problem. Given a subset $A$ of demand points, i.e., a collections of pairs $(i, t), i=1, \ldots, N, t=1, \ldots, T$, let $D(A)=\sum_{(i, t) \in A} d_{i t}$ denote the cumulative demand of the set $A ; \ell_{A}=\left\lceil\frac{D(A)}{C}\right\rceil$ be the cover number of $A$, i.e., the minimum number of orders required to satisfy the demands in $A ; \lambda_{A}=\ell_{A} C-D(A)$; $R_{A}=C-\lambda_{A}$ be the residual capacity of $A$, i.e., the capacity required to satisfy the demands in $A$ after $\ell_{A}-1$ orders are fully used; and $r_{A}=R_{A} / C\left(=1-\ell_{A}+D(A) / C\right)$ be the fraction of the residual capacity. Observe that by definition $0<R_{A} \leq C$ and $0<r_{A} \leq 1$. Moreover, a subset $F$ of orders (i.e., $F \subseteq\{1, \ldots, T\})$ is called a cover of $A$ if $|F| \geq \ell_{A}$.

We claim that the following inequalities are valid when $F$ is a cover of $A$ :

$$
\sum_{(i, t) \in A} \sum_{s \in F} d_{i t} x_{s t}^{i}-R_{A} \sum_{s \in F} y_{s} \leq D(A)-\ell_{A} R_{A}
$$

(Note that by summing over $(i, t) \in A$ and $s \in F$, we actually refer only to combinations for which $s \leq t$.) The validity of Inequalities (7) in the multi-item capacitated lot-sizing problem can be obtained as a special case of the general mixed integer rounding inequalities, or in short MIR inequalities (see, e.g., Nemhauser and Wolsey [17]). An MIR inequality is defined with respect to the simple mixed-integer set $\mathcal{Q}=\{x \in R, y \in Z: x+y \geq b, x \geq 0\}$, for which it is easy to prove the validity of the inequality $x+\hat{b} y \geq \hat{b}\lceil b\rceil$, where $\lceil b\rceil$ is equal to $b$ rounded up to the next integer, and $\hat{b}=b-\lfloor b\rfloor$. Of course, the MIR inequality turns out to be useful if and only if $\hat{b}>0$, otherwise it trivially becomes $x \geq 0$. Moreover, the $y$ values of points in the convex hull of $\mathcal{Q}$ (i.e., the continuous relaxation of $\mathcal{Q}$ ) that violate the MIR inequality above fall within $(\lfloor b\rfloor,\lceil b\rceil)$. (It can be verified that the point $(\hat{b},\lfloor b\rfloor)$ is in $\mathcal{Q}$ and satisfies the MIR inequality, see Figure 1.) This can be generalized to more complicated sets that involve more variables, as long as the variables can be split into an integral part and a continuous nonnegative part. 
In particular, we apply an MIR derivation to the mixed-integer set whose defining inequality is:

$$
\frac{1}{C} \sum_{(i, t) \in A} \sum_{s \notin F} d_{i t} x_{s t}^{i}+\sum_{s \in F} y_{s} \geq \frac{D(A)}{C} .
$$

It is easy to see that Inequality (8) is valid for the system (1)-(6). Specifically, Constraint (2) implies that $D(A)=\sum_{(i, t) \in A} \sum_{s \in F} d_{i t} x_{s t}^{i}+\sum_{(i, t) \in A} \sum_{s \notin F} d_{i t} x_{s t}^{i}$; then replace the first term in the right hand side of the equality by an upper bound $C \sum_{s \in F} y_{s}$ (see Constraint (4)) and divide by $C$ to get the desired Inequality (8).

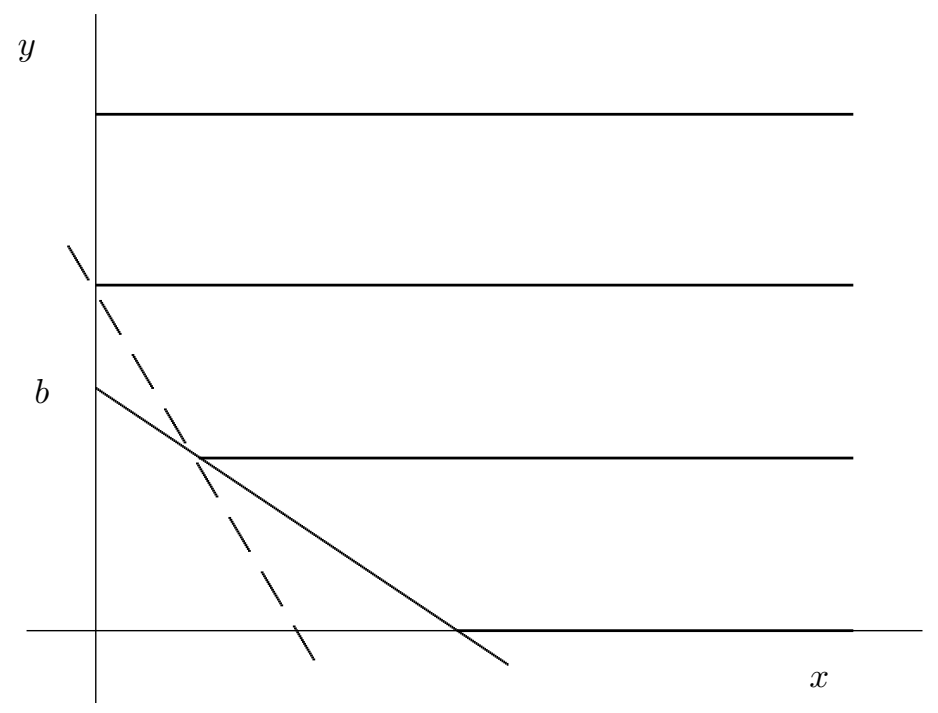

Figure 1: MIR derivation on the mixed-integer set $\mathcal{Q}=\{x \in R, y \in Z: x+y \geq b, x \geq 0\}$.

Thus, by applying an MIR derivation to (8) one obtains:

$$
\frac{1}{C} \sum_{(i, t) \in A} \sum_{s \notin F} d_{i t} x_{s t}^{i}+r_{A} \sum_{s \in F} y_{s} \geq r_{A} \ell_{A},
$$

which coincides with (7). (Multiply by $C$ and add $\sum_{(i, t) \in A} \sum_{s \in F} d_{i t} x_{s t}^{i}$.)

Separation To the best of our knowledge the complexity of separating flow-cover inequalities is unknown. Aardal [1] has shown that flow-cover inequalities can be separated in polynomial time for a fixed set of demand points via a simple greedy procedure.

Next we consider a fixed subset of orders $\bar{F} \subseteq\{1, \ldots, T\}$, and describe a polynomial time algorithm to separate flow-cover inequalities that correspond to the subset of orders $\bar{F}$. For the description of the algorithm, it will be useful to rewrite flow-cover inequalities that correspond to the set $\bar{F}$ as

$$
\sum_{(i, t) \in A} \frac{d_{i t}}{C}\left(1-\sum_{s \in \bar{F}} x_{s t}^{i}\right) \geq r_{A}\left(\ell_{A}-\sum_{s \in \bar{F}} y_{s}\right) .
$$

Observe that (10) above may still contain exponentially many constraints, one for each subset $A$ of demand points that can be covered by $\bar{F}$. However, this is similar to the residual capacity inequalities introduced by Magnanti, Marchandani and Vachani [16] for another mixed-integer set called the splittable flow arc set $X=\left\{x \in R^{n}, y \in Z: \sum_{i=1}^{n} a_{i} x_{i} \leq a_{0}+y, x_{i} \geq 0, x_{i} \leq 1, i=1, \ldots, n, y \in\{0,1\}\right\}$. It has been shown that residual capacity inequalities are sufficient to characterize conv $(X)[16,5]$. Moreover, Atamtürk and Rajan [5] have described an $O(n)$ time separation algorithm for residual capacity inequalities.

Building on the results of Atamtürk and Rajan [5], we can state and prove the following theorem.

THEOREM 3.1 Consider a subset of orders $\bar{F}$. Then there exists a polynomial time separation algorithm for the inequalities in (10). The algorithm runs in $O\left(N T^{2}\right)$ time. 
Proof. Consider a fractional solution $(\hat{x}, \hat{y})$ of the LP relaxation defined by (1)-(6) and (10). Then the separation problem for (10) can be formulated as a nonlinear mixed-integer program:

$$
\min \sum_{i=1}^{N} \sum_{t=1}^{T} \frac{d_{i t}}{C}\left(1-\sum_{s \in \bar{F}} \hat{x}_{s t}^{i}\right) z_{t}^{i}-r_{A}\left(\ell_{A}-\sum_{s \in \bar{F}} \hat{y}_{s}\right)
$$

subject to

$$
\begin{aligned}
\sum_{i=1}^{N} \sum_{t=1}^{T} \frac{d_{i t}}{C} z_{t}^{i} & =\left(\ell_{A}-1\right)+r_{A}, \\
z_{t}^{i} & \in\{0,1\} \\
r_{A} & \in(0,1), \\
\ell_{A} & \text { integer. }
\end{aligned}
$$

By definition $r_{A}>0$. Also observe that for an Inequality (10) to be violated, it must be that $r_{A}<1$. Indeed, in the case $r_{A}=1$ Inequalities (10) are implied by Inequalities (4) of the model. Since Inequality (9) is an MIR inequality applied to (8) it follows that it can be violated only if $\ell_{A}-1<\sum_{s \in \bar{F}} \hat{y}_{s}<\ell_{A}$. (Note that $\ell_{A}$ is equivalent to $\lceil b\rceil$ used in the definition of MIR inequalities above.) Using the observation that $\ell_{A}=\left\lceil\sum_{s \in \bar{F}} \hat{y}_{s}\right\rceil$ and Equation (12) to substitute out $\ell_{A}$ and $r_{A}$, the mixed-integer nonlinear program can be reformulated as a binary MIP. Now use the identity $1-\left\lceil\sum_{s \in \bar{F}} \hat{y}_{s}\right\rceil=-\left\lfloor\sum_{s \in \bar{F}} \hat{y}_{s}\right\rfloor$ to get the following separation MIP:

$\min \sum_{i=1}^{N} \sum_{t=1}^{T} \frac{d_{i t}}{C} z_{t}^{i}\left(-\sum_{s \in \bar{F}} \hat{x}_{s t}^{i}-\left\lfloor\sum_{s \in \bar{F}} \hat{y}_{s}\right\rfloor+\sum_{s \in \bar{F}} \hat{y}_{s}\right)+\left(\left\lceil\sum_{s \in \bar{F}} \hat{y}_{s}\right\rceil-\sum_{s \in \bar{F}} \hat{y}_{s}\right)\left\lfloor\sum_{s \in \bar{F}} \hat{y}_{s}\right\rfloor$

subject to

$$
\begin{aligned}
& \sum_{i=1}^{N} \sum_{t=1}^{T} \frac{d_{i t}}{C} z_{t}^{i}<\left\lceil\sum_{s \in \bar{F}} \hat{y}_{s}\right\rfloor, \\
& \sum_{i=1}^{N} \sum_{t=1}^{T} \frac{d_{i t}}{C} z_{t}^{i}>\left\lfloor\sum_{s \in \bar{F}} \hat{y}_{s}\right\rfloor, \\
& z_{t}^{i} \in\{0,1\}
\end{aligned}
$$

Note that (17) and (18) are equivalent to (12) above. Also observe that the second part of the objective function of the above MIP is a nonnegative constant. Moreover an inequality in (10) is violated if and only if the optimal value of the above MIP is strictly negative.

The following simple algorithm solves the separation MIP to optimality in $O\left(N T^{2}\right)$ time:

(i) Construct the set $\widetilde{A}=\left\{(i, t): \sum_{s \in \bar{F}} \hat{x}_{s t}^{i}>\sum_{s \in \bar{F}} \hat{y}_{s}-\left\lfloor\sum_{s \in \bar{F}} \hat{y}_{s}\right\rfloor, i=1, \ldots, N, t=1, \ldots, T\right\}$;

(ii) Set $z_{t}^{i}=1, \forall(i, t) \in \widetilde{A}$ and 0 otherwise;

(iii) If the objective function (16) is negative and Constraints (17)-(18) are satisfied for $z$ above, then set $\widetilde{A}$ defines a violated Inequality (10), otherwise no violated inequality exists.

Next we prove the correctness of the above algorithm. Observe that the solution corresponding to the set $\widetilde{A}$ provides a lower bound on the optimal value of the separation MIP above. Thus, if $\widetilde{A}$ is feasible with respect to (17) and (18) there is nothing to prove. It is then sufficient to show that if $\widetilde{A}$ is not feasible, there is no violated constraint in (10).

First consider the case $\sum_{(i, t) \in \widetilde{A}} \frac{d_{i t}}{C} \leq\left\lfloor\sum_{s \in \bar{F}} \hat{y}_{s}\right\rfloor$. We bound the objective value of the solution 
corresponding to $\widetilde{A}$ (in all the inequalities below when we write $\sum_{s \in \bar{F}} \hat{x}_{s t}^{i}$, we refer only to $s \leq t$ ),

$$
\begin{aligned}
\sum_{(i, t) \in \tilde{A}} d_{i t} / C & \left.\left(-\sum_{s \in \bar{F}} \hat{x}_{s t}^{i}-\left\lfloor\sum_{s \in \bar{F}} \hat{y}_{s}\right\rfloor+\sum_{s \in \bar{F}} \hat{y}_{s}\right)+\left(\left\lceil\sum_{s \in \bar{F}} \hat{y}_{s}\right\rfloor-\sum_{s \in \bar{F}} \hat{y}_{s}\right) \mid \sum_{s \in \bar{F}} \hat{y}_{s}\right\rfloor \\
& \geq \sum_{(i, t) \in \tilde{A}} d_{i t} / C\left(-\sum_{s \in \bar{F}} \hat{x}_{s t}^{i}-\left\lfloor\sum_{s \in \bar{F}} \hat{y}_{s}\right\rfloor+\sum_{s \in \bar{F}} \hat{y}_{s}\right)+\left(\left\lceil\sum_{s \in \bar{F}} \hat{y}_{s}\right\rfloor-\sum_{s \in \bar{F}} \hat{y}_{s}\right) \sum_{(i, t) \in \widetilde{A}} d_{i t} / C \\
& =\sum_{(i, t) \in \tilde{A}} d_{i t} / C\left(-\sum_{s \in \bar{F}} \hat{x}_{s t}^{i}+\left\lfloor\sum_{s \in \bar{F}} \hat{y}_{s}\right\rfloor-\left\lfloor\sum_{s \in \bar{F}} \hat{y}_{s}\right\rfloor\right)=\sum_{(i, t) \in \widetilde{A}} d_{i t} / C\left(1-\sum_{s \in \bar{F}} \hat{x}_{s t}^{i}\right) \geq 0 .
\end{aligned}
$$

The first inequality follows from the assumption that $\sum_{(i, t) \in \tilde{A}} \frac{d_{i t}}{C} \leq\left\lfloor\sum_{s \in \bar{F}} \hat{y}_{s}\right\rfloor$, and the last inequality follows from the Constraint (2).

Consider now the case $\sum_{(i, t) \in \tilde{A}} \frac{d_{i t}}{C} \geq\left\lceil\sum_{s \in \bar{F}} \hat{y}_{s}\right\rceil$. We again bound the objective of the solution corresponding to the set $\widetilde{A}$,

$$
\begin{aligned}
\sum_{(i, t) \in \widetilde{A}} d_{i t} / C\left(-\sum_{s \in \bar{F}} \hat{x}_{s t}^{i}-\left\lfloor\sum_{s \in \bar{F}} \hat{y}_{s}\right\rfloor+\sum_{s \in \bar{F}} \hat{y}_{s}\right)+\left(\left\lceil\sum_{s \in \bar{F}} \hat{y}_{s}\right\rceil-\sum_{s \in \bar{F}} \hat{y}_{s}\right)\left\lfloor\sum_{s \in \bar{F}} \hat{y}_{s}\right\rfloor \\
\left.\geq-\sum_{(i, t) \in \widetilde{A}} d_{i t} / C \sum_{s \in \bar{F}} \hat{x}_{s t}^{i}+\left\lceil\sum_{s \in \bar{F}} \hat{y}_{s}\right\rfloor\left(-\left\lfloor\sum_{s \in \bar{F}} \hat{y}_{s}\right\rfloor+\sum_{s \in \bar{F}} \hat{y}_{s}\right)+\left(\left\lceil\sum_{s \in \bar{F}} \hat{y}_{s}\right\rceil-\sum_{s \in \bar{F}} \hat{y}_{s}\right) \mid \sum_{s \in \bar{F}} \hat{y}_{s}\right\rfloor \\
=-\sum_{(i, t) \in \widetilde{A}} d_{i t} / C \sum_{s \in \bar{F}} \hat{x}_{s t}^{i}+\sum_{s \in \bar{F}} \hat{y}_{s} \geq 0 .
\end{aligned}
$$

The first inequality follows from the assumption that $\sum_{(i, t) \in \tilde{A}} \frac{d_{i t}}{C} \geq\left\lceil\sum_{s \in \bar{F}} \hat{y}_{s}\right\rceil$, the first equality follows from the identity $\left\lfloor\sum_{s \in \bar{F}} \hat{y}_{s}\right\rfloor=\left\lceil\sum_{s \in \bar{F}} \hat{y}_{s}\right\rceil-1$, and the last inequality follows from Constraints (4). This concludes the proof of the theorem.

An LP Next we describe an LP based on (1)-(5), relaxation of the integrality constraint of (6) and a subset of the flow-cover inequalities defined in (7) above.

Let $\mathcal{F}:=\{[s, t]: 1 \leq s \leq t \leq T\}$ be the collection of all subsets of orders defined by intervals $[s, t]$. Consider the LP defined by (1)-(5), the relaxation of (6) and only the inequalities in (7) that correspond to subsets of orders $F \in \mathcal{F}$. Recall that in Theorem 3.1 we have shown that flow-cover inequalities that correspond to a fixed subset of orders can be separated in polynomial time. Since the cardinality of the set $\mathcal{F}$ is $O\left(T^{2}\right)$, it follows that the above LP can be solved to optimality in polynomial time by using the Ellipsoid method. Let $(\hat{x}, \hat{y})$ be the optimal solution of that LP and $V_{L P}$ be the respective optimal value. Since the LP is a relaxation of the problem, it follows that $V_{L P}$ is a lower bound on the optimal cost denoted by $V_{O P T}$.

4. The Random-Shift Algorithm with Median Demand Assignment In this section, we describe an approximation algorithm for the capacitated multi-item lot-sizing problem with hard capacities that is based on the linear programming relaxation defined above by (1)-(5), the relaxation of the integrality constraint of (6) and the flow-cover inequalities in (7) that correspond to the collection of subsets $\mathcal{F}$ defined above.

We shall first show how to round the optimal fractional solution $(\hat{x}, \hat{y})$ of this LP to a feasible integer solution with cost that is at most twice $V_{L P}$. Since $V_{L P}$ is a lower bound on the optimal cost, this implies that the algorithm is a 2-approximation. In addition, we shall describe an on-the-fly variant of the algorithm that does not require adding all the respective flow-cover inequalities a-priori, but instead adds violated constraints on-the-fly until a (good) integer solution is obtained.

First, we present a randomized rounding procedure that we call Random-Shift with Median Assignment. This procedure rounds the fractional optimal solution $(\hat{x}, \hat{y})$ to a feasible integer solution $(\tilde{x}, \tilde{y})$ with expected cost that is at most twice the optimal cost $V_{O P T}$. We then discuss how to derandomize the algorithm, and get a deterministic 2-approximation algorithm. 
The rounding algorithm runs in two phases. In the first phase of the algorithm, we determine in which periods to place orders. Based on the outcome of the first phase of the algorithm, we decide how to assign demand points to orders.

4.1 Phase I: The Random-Shift Procedure We first describe Phase I of the algorithm which we call the Random-Shift procedure. This is similar in spirit to the work of Levi, Roundy, Shmoys and Sviridenko [15] on the single-warehouse and multi-retailer problem. In this phase we decide in which periods to place orders. This simple randomized procedure is based on the values $\hat{y}_{1}, \ldots, \hat{y}_{T}$. For each $s=1, \ldots, T$, let $\bar{y}_{s}=\min \left\{2 \hat{y}_{s}, 1\right\}$, i.e., we double the original value of each variable $\hat{y}_{s} \leq 0.5$ and make it equal to 1 if $\hat{y}_{s}>0.5$. We call $\hat{y}_{s}$ and $\bar{y}_{s}$ the fractional order and scaled fractional order in period $s=1, \ldots, T$, respectively. Next we shall use the values $\bar{y}_{1}, \ldots, \bar{y}_{T}$ to determine the periods in which orders are placed.

For the description of the Random-Shift procedure, consider the interval $\left(0, \sum_{s=1}^{T} \bar{y}_{s}\right]$, which corresponds to the total weight of scaled fractional orders. Each period $r=1, \ldots, T$ is then associated with the corresponding interval $\left(\sum_{s=1}^{r-1} \bar{y}_{s}, \sum_{s=1}^{r} \bar{y}_{s}\right]$, which is of length $\bar{y}_{r}$. In particular, some periods can correspond to empty intervals of length 0 (if $\hat{y}_{t}=\bar{y}_{t}=0$ ). The input for this procedure is a shift-parameter $\alpha$ that is chosen uniformly at random in $(0,1]$. Let $W$ be the smallest integer that is greater than or equal to $\sum_{s=1}^{T} \bar{y}_{s}$. Specifically, $W$ is the upper ceiling of the total cumulative weight of the scaled fractional orders; that is, $W=\left\lceil\sum_{s=1}^{T} \bar{y}_{s}\right\rceil$. Note that the interval $\left(0, \sum_{s=1}^{T} \bar{y}_{s}\right]$ is contained in the interval $[0, W]$. Within the interval $[0, W]$ focus on the sequence of points $0,1, \ldots, W-1$. The shift-parameter $\alpha$ induces a sequence of what we call shift-points. Specifically, the set of shift-points is defined as $\{\alpha+w: w=0, \ldots, W-1\}$. This set is constructed through a shift of random length $\alpha$ to the right of the points $0,1, \ldots, W-1$. Thus, there are $W$ shift-points that are all located within the interval $[0, W]$. Observe that the sequence of shift-points is a-priori random and is realized with the shift-parameter $\alpha$.

The shift-points determine the periods in which orders are placed. For each period $r=1, \ldots, T$, we place an order in that period if there is a shift-point within the interval $\left(\sum_{s=1}^{r-1} \bar{y}_{s}, \sum_{s=1}^{r} \bar{y}_{s}\right]$ that is associated with period $r$. That is, we place an order in period $r$ if for some integer $0 \leq w \leq W-1$ there exists a shift- point $\alpha+w$ that falls within the interval $\left(\sum_{s=1}^{r-1} \bar{y}_{s}, \sum_{s=1}^{r} \bar{y}_{s}\right]$. Let $\mathcal{T}:=\left\{r_{1}<r_{2}<\ldots<r_{Q}\right\}$ be the set of periods of the orders as determined in the first phase of the algorithm using the random-shift procedure. We set $\tilde{y}_{r_{m}}=1$ for each $m=1, \ldots, Q$, and call $r_{1}, \ldots, r_{Q}$ the opened orders.

Next we bound the expected ordering cost incurred by the random shift procedure.

LEMmA 4.1 Consider the Random-Shift procedure described above. Then, for each period $r=1, \ldots, T$, the probability of placing an order in period $r$ is at most $\bar{y}_{r} \leq 2 \hat{y}_{r}$. Thus, the total expected ordering cost of the Random-Shift procedure, denoted by $\mathcal{K}$, is at most twice the total ordering costs in the optimal LP solution. That is, $\mathcal{K} \leq \sum_{s=1}^{T} \bar{y}_{s} K_{s} \leq 2 \sum_{s=1}^{T} \hat{y}_{s} K_{s}$.

Proof. The shift-points $\{\alpha+w: w=0, \ldots, W-1\}$ are located over the interval $[0, W]$, and the distance between each two consecutive shift-points is 1 . Thus, for each period $r$ with $\hat{y}_{r} \geq 0.5$, there is one shift-point within the interval $\left(\sum_{s=1}^{r-1} \bar{y}_{s}, \sum_{s=1}^{r} \bar{y}_{s}\right]$, with probability 1 , since it is of length $\bar{y}_{r}=1$. This implies that, with probability 1 , there will be an order placed in period $r$. Since indeed $\bar{y}_{r}=1 \leq 2 \hat{y}_{r}$, the claim for this case follows.

Consider now a period $r$ with $\hat{y}_{r}<0.5$. There are exactly two possible subcases:

Case 1: The interval $\left(\sum_{s=1}^{r-1} \bar{y}_{s}, \sum_{s=1}^{r} \bar{y}_{s}\right]$ is contained in an interval $(w,(w+1)]$, for some integer $0 \leq w \leq$ $W-1$. Moreover, since the distance between consecutive shift-points is 1 , there is exactly one shift-point within the interval $(w,(w+1)]$; specifically, the shift-point $\alpha+w$. Moreover, this shift-point is uniformly distributed over the interval $(w,(w+1)]$. It follows that the probability that this shift-point falls within the interval $\left(\sum_{s=1}^{r-1} \bar{y}_{s}, \sum_{s=1}^{r} \bar{y}_{s}\right]$ is $\bar{y}_{r}=2 \hat{y}_{r}$. The claim for this case then follows.

Case 2: If Case 1 above does not apply, it must be the case that some point $w$ (where again $0 \leq w \leq W-1$ ) falls within the interval $\left(\sum_{s=1}^{r-1} \bar{y}_{s}, \sum_{s=1}^{r} \bar{y}_{s}\right]$. (Note that since $\bar{y}_{r}=2 \hat{y}_{r}<1$ there can be at most one such point.) Thus, the interval $\left(\sum_{s=1}^{r-1} \bar{y}_{s}, \sum_{s=1}^{r} \bar{y}_{s}\right]$ is split between the two intervals $(w-1, w]$ and $(w, w+1]$. Consider now the two intervals $\left(\sum_{s=1}^{r-1} \bar{y}_{s}, w\right]$ and $\left(w, \sum_{s=1}^{r} \bar{y}_{s}\right]$. Since the respective shift-points $\alpha+(w-1)$ and $\alpha+w$ are at distance 1 of each other, it follows that at most one of the intervals $\left(\sum_{s=1}^{r-1} \bar{y}_{s}, w\right]$ and 
$\left(w, \sum_{s=1}^{r} \bar{y}_{s}\right]$ above can contain a shift- point. Thus, the probability that there is a shift-point within the interval $\left(\sum_{s=1}^{r-1} \bar{y}_{s}, \sum_{s=1}^{r} \bar{y}_{s}\right]$ can be expressed as the sum of the probability of having a shift-point within the interval $\left(\sum_{s=1}^{r-1} \bar{y}_{s}, w\right]$ plus the probability of having a shift-point within the interval $\left(w, \sum_{s=1}^{r} \hat{y}_{s}\right]$ (since these are two disjoint events). Finally, by applying the arguments of Case 1 to each of the intervals $\left(\sum_{s=1}^{r-1} \bar{y}_{s}, w\right]$ and $\left(w, \sum_{s=1}^{r} \bar{y}_{s}\right]$, respectively, the claim for this case follows. This concludes the proof of the lemma.

Given the opened orders $r_{1}, \ldots, r_{Q}$, we can compute the cheapest assignments of demand points to opened orders by solving the corresponding transportation problem. The solution of the transportation problem will determine the values of $\tilde{x}_{s t}^{i}$, for each $(i, t)$ and $s \leq t$. However, it is not clear a-priori that the induced transportation problem has a feasible solution, and even if it has one, there is a question regarding the cost of this solution. Next we shall show that the induced transportation problem indeed has a feasible solution with cost denoted by $\mathcal{H}$ that is at most twice the holding cost incurred by the optimal fractional solution $(\hat{x}, \hat{y})$. That is, the holding cost incurred by the algorithm is $\mathcal{H} \leq 2 \sum_{i=1}^{N} \sum_{t=1}^{T} \sum_{s=1}^{t} H_{s t}^{i} \hat{x}_{s t}^{i}$.

4.2 Phase II: The Median Assignment Next we describe a constructive procedure, called the Median Assignment, that assigns all the demand points to the opened orders $r_{1}, \ldots, r_{Q}$, and incurs a holding cost that is at most twice the holding cost incurred by the optimal fractional solution $(\hat{x}, \hat{y})$. Observe that the optimal solution to the transportation problem induced by the opened orders $r_{1}, \ldots, r_{Q}$ incurs no greater holding cost. To describe the procedure we introduce the notion of flow-requirements of demand point $(i, t)$. Focus on a specific demand point $(i, t)$, and let $s_{1}<s_{2}<\cdots<s_{G}$ be the fractional orders that fractionally serve this demand point in the optimal LP solution $(\hat{x}, \hat{y})$. In particular, $\hat{x}_{s_{g}, t}^{i}>0$, for each $g=1 \ldots, G$, and $\sum_{g=1}^{G} \hat{x}_{s_{g}, t}^{i}=1$. Let $s_{M}$ be the median order of $(i, t)$, i.e., the latest point in time such that at least half of the demand $d_{i t}$ is satisfied from orders within $\left[s_{M}, t\right]$. That is, $M=\max \left\{m: \sum_{g=m}^{G} \hat{x}_{s_{g}, t}^{i} \geq 0.5\right\}$. For each $g=1, \ldots, G$, let $z_{s_{g}, t}^{i}$ be the flow-requirement of $(i, t)$ that is due to $s_{g}$. Specifically, for each $g=1, . ., M-1$, we define $z_{s_{g}, t}^{i}=2 \hat{x}_{s_{g}, t}^{i} d_{i t}$; for $g=M$ we define $z_{s_{M}, t}^{i}=2\left(0.5-\sum_{q=1}^{M-1} \hat{x}_{s_{q}, t}^{i}\right) d_{i t} ;$ and for each $g=M+1, \ldots, G$, we define $z_{s_{g}, t}^{i}=0$.

Note that the flow-requirements defined above do not necessarily provide a feasible assignment of demands to orders. Intuitively, we consider the median order that splits the assignment of demand point $(i, t)$ in the optimal fractional solution $(\hat{x}, \hat{y})$ into two equal halves. We then ignore the upper (later) half and scale the lower (earlier) half by 2 . However, we shall use the flow-requirements $z_{s_{1}, t}^{i}, \ldots, z_{s_{G}, t}^{i}$ to construct a feasible assignment of demands with relatively low holding costs. First, observe that $\sum_{g=1}^{G} z_{s_{g}, t}^{i}=d_{i t}$. We wish to construct a feasible assignment that, for each demand point $(i, t)$ and order $s_{g}$, satisfies at least $z_{s_{g}, t}^{i}$ units of $d_{i t}$ from orders within the interval $\left[s_{g}, t\right]$. That is, the flow-requirement $z_{s_{g}, t}^{i}$ is satisfied either from $s_{g}$ or from orders later in time. We will say that such an assignment satisfies all the flow-requirements. (Recall that $s_{g}$ and $z_{s_{g}, t}^{i}$ are specific to demand point $(i, t)$ based on the optimal fractional solution $(\hat{x}, \hat{y})$.)

Consider any assignment of demands that satisfies all the flow-requirements of all demands. Since the assignment satisfies $z_{s_{g}, t}^{i}$ units of $d_{i t}$ either from $s_{g}$ or from orders even later in time, we conclude that the holding cost incurred by each demand point $(i, t)$ is at most $\sum_{g=1}^{G} z_{s_{g}, t}^{i} h_{s_{g}, t}^{i}$. However, by the definition of the flow-requirements, we have

$$
\sum_{g=1}^{G} z_{s_{g}, t}^{i} h_{s_{g}, t}^{i} \leq 2 \sum_{g=1}^{G} \hat{x}_{s_{g}, t}^{i} d_{i t} h_{s_{g}, t}^{i}=2 \sum_{g=1}^{G} \hat{x}_{s_{g}, t}^{i} H_{s_{g}, t}^{i} .
$$

That is, the holding cost incurred is at most twice the holding costs incurred by $(i, t)$ in $(\hat{x}, \hat{y})$. In light of Lemma 4.1 above, if such an assignment exists, the resulting feasible solution $(\tilde{x}, \tilde{y})$ has cost that is at most twice the optimal value of the LP $V_{L P}$. Since $V_{L P}$ is a lower bound on the optimal cost, it follows that the cost of the solution is at most twice the optimal cost. It is then left to show that such an assignment does exist. Next we shall describe the details of the Median Assignment procedure. (A summary of the Median Assignment algorithm can be found below.)

We construct the Median Assignment in stages indexed by $\tau=T, \ldots, 1$. In each stage $\tau$, we consider the set of positive flow-requirements due within $\tau$, i.e., the set $\mathcal{B}_{\tau}=\left\{z_{\tau t}^{i}>0: i=1, \ldots, N, t=\tau, \ldots, T\right\}$. These are the flow-requirements that need to be satisfied from orders within $[\tau, T]$. Partition the set $\mathcal{B}_{\tau}$ into sets $\mathcal{B}_{\tau t}$, for $t=\tau, \ldots, T$, where $\mathcal{B}_{\tau t}=\left\{z_{\tau t}^{i}>0: i=1, \ldots, N\right\}$. We then consider the sets $\mathcal{B}_{\tau t}$ 
in decreasing order $t=T, \ldots, \tau$. For each flow-requirement $z_{\tau t}^{i} \in \mathcal{B}_{\tau t}$, we consider the opened orders (decided upon in Phase I) within $[\tau, t]$ in decreasing order from latest to earliest. The flow-requirement $z_{\tau t}^{i}$ is then assigned to these orders greedily according to the current available capacity. More rigorously, consider a specific flow-requirement $z_{\tau t}^{i}$ and let $\mathcal{T}_{[\tau, t]}=\mathcal{T} \cap[\tau, t]=\left\{e_{1}<e_{2}<\cdots<e_{V}\right\}$ be the set of opened orders within the interval $[\tau, t]$. Let $\delta_{V}=\min \left\{z_{\tau t}^{i},\left(C-\sum_{j=1}^{N} \sum_{u=e_{V}}^{T} \tilde{x}_{e_{V}, u}^{j} d_{j u}\right)^{+}\right\}$, and for each $v=V-1, \ldots, 1$, let $\delta_{v}=\min \left\{\left(z_{\tau t}^{i}-\sum_{q=v+1}^{V} \delta_{q}\right)^{+},\left(C-\sum_{j=1}^{N} \sum_{u=e_{v}}^{T} \tilde{x}_{e_{v}, u}^{j} d_{j u}\right)^{+}\right\}$. (Recall that $(x)^{+}=\max \{x, 0\}$.) We then update $\tilde{x}_{e_{v}, t}^{i}=\tilde{x}_{e_{v}, t}^{i}+\delta_{v} / d_{i t}$, for each $v=1, \ldots, V$.

\section{The Median Assignment Algorithm - Summary:}

(i) Initialization: $\tau=T$; for each $i, s \leq t$, set $\tilde{x}_{s t}^{i}=0$; for each $s \in \mathcal{T}$ set $\tilde{y}_{s}=1$, otherwise set $\tilde{y}=0$; set the capacity of order $s$ to be $C_{s}=\tilde{y}_{s} C$

(ii) Assignment:

(i) If $\tau=0$ terminate and declare 'success', otherwise set $k=T$ and go to (iii)

(ii) If $k<\tau$, set $\tau=\tau-1$ and go to $(i)$

(iii) If all the positive flow-requirements in $\mathcal{B}_{\tau k}$ are satisfied, set $k=k-1$ and go to (ii), otherwise choose a positive flow-requirement $z_{\tau k}^{i} \in \mathcal{B}_{\tau k}$ not yet satisfied, set $v=z_{\tau k}^{i}, s=k$ and go to (iv)

(iv) While $v>0$

- if $s<\tau$ terminate and declare 'failure'

- Set $\tilde{x}_{s k}^{i}=\tilde{x}_{s k}^{i}+\min \left\{v, C_{s}\right\} / d_{i k} ; v=v-\tilde{x}_{s k}^{i} d_{i k} ; C_{s}=C_{s}-\tilde{x}_{s k}^{i} d_{i k}$

- Set $s=s-1$

(v) Go to $($ iii)

By construction it follows that if completed successfully, the Median Assignment described above satisfies the flow-requirements of all demand points. Thus, to establish a bound on the holding cost incurred by the algorithm, it is sufficient to show that the Median Assignment can be completed successfully.

Before we prove that, we would like to state a technical lemma that draws a connection between the cumulative fractional orders opened by the fractional solution $(\hat{x}, \hat{y})$, and the corresponding number of integral orders opened in Phase I of the algorithm.

Lemma 4.2 Consider the interval $[s, t]$ for some $s \leq t$, and suppose that the cumulative fractional orders opened in $[s, t]$ by the LP optimal solution $(\hat{x}, \hat{y})$ is equal $L+\beta$, where $L$ is a non-negative integer and $\beta$ is between 0 and 1. That is, $\sum_{u=s}^{t} \hat{y}_{u}=L+\beta$. Then if $\beta \geq 0.5$, the number of orders placed in Phase $I$ of the algorithm over the interval $[s, t]$ is at least $L+1$. That is, $\sum_{u=s}^{t} \tilde{y}_{u} \geq L+1$.

ProOF. First, observe that by the construction of the Random-Shift procedure there will be at least $L$ opened orders over the interval $[s, t]$, regardless of what the value of $\beta$ is. Moreover, the number of orders opened over the interval $[s, t]$ is at least $\left\lfloor\sum_{u=s}^{t} \bar{y}_{u}\right\rfloor$, which is the floor of the cumulative weight of scaled fractional orders over the interval. Thus, it is sufficient to show that if $\sum_{u=s}^{t} \hat{y}_{u}=L+\beta$ and $\beta \geq 0.5$, then $\sum_{u=s}^{t} \bar{y}_{u} \geq L+1$.

Let $\mathcal{O}$ be set of periods $u$ in $[s, t]$ for which $\hat{y}_{u} \geq 0.5$. If $|\mathcal{O}| \geq L+1$, then there is nothing to prove since, for each $u \in \mathcal{O}$, we have $\bar{y}_{u}=1$. Assume now that $|\mathcal{O}| \leq L$. By the construction of the scaled fractional orders we have

$$
\begin{aligned}
\sum_{u=s}^{t} \bar{y}_{u} & =|\mathcal{O}|+2 \sum_{u \in[s, t] \backslash \mathcal{O}} \hat{y}_{u} \\
& =|\mathcal{O}|+2\left(L+\beta-\sum_{u \in \mathcal{O}} \hat{y}_{u}\right) \geq 2 L-|\mathcal{O}|+2 \beta \\
& \geq L+2 \beta \geq L+1 .
\end{aligned}
$$

The second equality follows from the fact that $\sum_{u=s}^{t} \hat{y}_{u}=L+\beta$. The first inequality follows from the fact that $\sum_{u \in \mathcal{O}} \hat{y}_{u} \leq|\mathcal{O}|$. The second inequality follows from the assumption that $|\mathcal{O}| \leq L$. Finally, the last inequality follows from the assumption that $\beta \geq 0.5$. The proof of the lemma then follows. 
Lemma 4.3 The Median Assignment can be completed successfully.

Proof. Assume by contradiction that the Median Assignment cannot be completed at some stage $\tau$ due lack of capacity to satisfy the flow-requirement $z_{\tau, \bar{t}}^{i}$ of some demand point $(i, \bar{t})$. It follows that all the opened orders within the interval $[\tau, \bar{t}]$ are currently fully used by the integer partial solution $(\tilde{x}, \tilde{y})$. That is, for each $r \in \mathcal{T} \cap[\tau, \bar{t}]$, we have $\sum_{i=1}^{N} \sum_{u=r}^{T} \tilde{x}_{r, u}^{i} d_{i u}=C$. Now let $\bar{r}$ be the earliest opened order within $(\bar{t}, T] \cap \mathcal{T}$ that still has free capacity or $T+1$ if no such order exists. That is,

$$
\bar{r}=\min \left\{\arg \min \left\{r \in \mathcal{T} \cap(\bar{t}, T]: \sum_{i=1}^{N} \sum_{u=r}^{T} \tilde{x}_{r, u}^{i} d_{i u}<C\right\}, T+1\right\} .
$$

Let $F=[\tau, \bar{r})$ be the corresponding interval of orders.

Next we focus on the set of demand points $(i, u)$ with positive flow-requirements that are due within $[\tau, \bar{r})$, i.e., the set of demand points $A=\left\{(i, t): t \in[\tau, \bar{r})\right.$ and $\left.\sum_{s=\tau}^{t} z_{s t}^{i}>0\right\}$. Using the notation in Section 3 we write $D(A)=\sum_{(i, t) \in A} d_{i t}=\left(\ell_{A}-1\right) C+R_{A}$, where $\ell_{A} \geq 1$ is an integer and $0<R_{A} \leq C$. Consider again the integer partial solution $(\tilde{x}, \tilde{y})$ at the moment the Median Assignment terminated due to lack of capacity. Recall that $\bar{r}=T+1$ or $\bar{r} \in \mathcal{T}$ is an opened order with free capacity. By the construction of the Median Assignment it follows that no demand point outside the interval $[\tau, \bar{r})$ is being served by the partial solution $(\tilde{x}, \tilde{y})$ from orders within the interval. That is, $\tilde{x}_{s t}^{i}=0$ for each $(i, t)$ with $t \geq \bar{r}$ and $s \in[\tau, \bar{r})$. This implies that all the available capacity of the opened orders within the interval $[\tau, \bar{r})$ is fully used by the integer partial solution $(\tilde{x}, \tilde{y})$ to serve only demand points in $A$. (It can be verified that any demand point $(i, t)$ with $t \in[\tau, \bar{r})$ but not in $A$, is not being served in $(\tilde{x}, \tilde{y})$ from within the interval $[\tau, \bar{r})$ since it does not have any positive flow-requirements that are due within this interval.) Moreover, since the Median Assignment could have not been completed, it follows that the flow-requirements of demand points in $A$ that are due within the interval $F$ exceed the total opened capacity over $F$. That is,

$$
\sum_{(i, t) \in A} \sum_{u \in F} z_{u t}^{i}>\sum_{u \in F} \tilde{y}_{u} C
$$

Now consider the set of fractional orders in the optimal LP solution $(\hat{x}, \hat{y})$ over $F$. Let $\sum_{u \in F} \hat{y}_{u} C=$ $(L-1) C+R$, where $L \geq 1$ is a nonnegative integer and $0<R \leq C$. The rest of the proof is based on comparing $\ell_{A}$ and $R_{A}$ to $L$ and $R$, respectively, and deriving a contradiction.

We first claim that $L \leq \ell_{A}$. This follows from the fact that

$$
C \cdot(L-1) \leq \sum_{u \in F} \tilde{y}_{u} C<\sum_{(i, t) \in A} \sum_{u \in F} z_{u t}^{i} \leq D(A) \leq \ell_{A} \cdot C .
$$

We have already seen in the proof of Lemma 4.2 that since $\sum_{u \in F} \hat{y}_{u} \geq L-1$ the Random-Shift procedure will open at least $L-1$ orders over the interval $F$, i.e., $\sum_{u \in F} \tilde{y}_{u} C \geq(L-1) C$. However, Inequality (20) implies that $\sum_{(i, t) \in A} \sum_{u \in F} z_{u t}^{i}>\tilde{y}_{u} C \geq(L-1) C$. Finally, observe that the overall flow-requirements of demand points in $A$ cannot exceed $D(A)$, which is at most $\ell_{A} \cdot C$. The proof of the claim then follows.

Next we claim that $R / C<0.5$. Assume otherwise. It follows that $\sum_{u \in F} \hat{y}_{u} \geq(L-1)+0.5$, and by Lemma 4.2 we conclude that there are at least $L$ opened orders over $F$. That is, $\sum_{u \in F} \tilde{y}_{u} C \geq L C$. However, the flow-requirements of a demand point $(i, t)$ over any interval $[\tau, t]$ are always bounded by the flow in the fractional optimal solution $(\hat{x}, \hat{y})$, sent to $(i, t)$ from orders within the interval. That is,

$$
\sum_{u \in F} \sum_{(i, t) \in A} z_{u t}^{i} \leq \sum_{u \in F} \sum_{(i, t) \in A} \hat{x}_{u, t}^{i} d_{i t} \leq \sum_{u \in F} \hat{y}_{u} C=(L-1) C+R .
$$

The first inequality follows from the fact that $F=[\tau, \bar{r})$ and the definition of the flow requirements. The last inequality follows from (4). It follows that a capacity of $L C$ units is sufficient to satisfy all the flow-requirements that are due within $F$ of all demand points in $A$, which leads to contradiction.

Next we claim that $L=\ell_{A}$. Assume otherwise, i.e., $\ell_{A}>L$. Since each demand point $(i, t) \in A$ has positive flow-requirements over $F$, it follows that $\sum_{u \in F} \hat{x}_{u, t}^{i}>0.5$. However, by the construction of the flow-requirements this implies that its total flow-requirements over $F$ can be expressed as

$$
\sum_{u \in F} z_{u t}^{i}=2 d_{i t}\left(\sum_{u \in F} \hat{x}_{u, t}^{i}-0.5\right) \text {. }
$$


Thus, the total flow-requirements of demand points in $A$ over $F$ can be expressed as

$$
\begin{aligned}
\sum_{(i, t) \in A} \sum_{u \in F} z_{u t}^{i} & =2\left(\sum_{(i, t) \in A} \sum_{u \in F} \hat{x}_{u, t}^{i} d_{i t}-0.5 D(A)\right) \\
& \leq 2\left(\sum_{u \in F} \hat{y}_{u} C-0.5 D(A)\right)=2(L-1) C+2 R-\left(\ell_{A}-1\right) C-R_{A} \\
& \leq(2 L-2+1-L+0) C \leq(L-1) C .
\end{aligned}
$$

The last inequality follows from the assumptions that $\ell_{A}-1 \geq L$ and that $2 R<C$. Moreover, this implies that capacity of $(L-1) C$ units is sufficient to satisfy all the flow-requirements that are due within $F$ of the demand points $(i, t) \in A$. However, we have already seen that there are at least $L-1$ opened orders over $F$, i.e., $\sum_{u \in F} \tilde{y}_{u} \geq L-1$. Since all of them are fully used to satisfy flow-requirements of demand points in $A$, this again leads to contradiction.

Suppose now that $R / C<0.5$ and $\ell_{A}=L$. This implies that the set of orders $F$ is a cover of the set of demands $A$. Moreover, $F \in \mathcal{F}$ (recall that $\mathcal{F}$ is the collection of all subsets of orders defined by intervals $[s, t])$ which implies that the solution $(\hat{x}, \hat{y})$ satisfies the flow-cover inequality that corresponds to $F$ and A. It follows that

$$
\begin{aligned}
D(A)-\sum_{(i, t) \in A} \sum_{u \in F} \hat{x}_{u, t}^{i} d_{i t} & \geq R_{A}\left(\ell_{A}-\sum_{u \in F} \hat{y}_{u}\right)=R_{A}\left(\ell_{A}-(L-1)-R / C\right) \\
& =R_{A}(1-R / C) \geq 0.5 R_{A} .
\end{aligned}
$$

The first inequality follows from the flow-cover inequality with respect to $F$ and $A$. The first equality follows from the fact that $\sum_{u \in F} \hat{y}_{u}=L-1+R / C$. The last inequality follows from the fact that $R / C<$ 0.5. We conclude that $2\left(D(A)-\sum_{(i, t) \in A} \sum_{u \in F} \hat{x}_{u, t}^{i} d_{i t}\right) \geq R_{A}$. However, $D(A)-\sum_{(i, t) \in A} \sum_{u \in F} \hat{x}_{u, t}^{i} d_{i t}$ is exactly the portion of $D(A)$ that is being served in the optimal fractional solution $(\hat{x}, \hat{y})$ from outside $F$. Moreover, we have already seen that, for each demand point $(i, t) \in A$, more than half of the demand $d_{i t}$ is served by $(\hat{x}, \hat{y})$ from within $F$, i.e., $\sum_{u \in F} \hat{x}_{u, t}^{i}>0.5$. By the construction of the flow-requirements this implies that the total flow-requirements of demand points $(i, t) \in A$ that are due outside $F$ is exactly $2\left(D(A)-\sum_{(i, t) \in A} \sum_{u \in F} \hat{x}_{u, t}^{i} d_{i t}\right) \geq R_{A}$. In turn, this implies that the total flow-requirements that are due within $F$ is at most $\left(\ell_{A}-1\right) C=(L-1) C$. However, as we have already seen, this leads to contradiction since there are at least $(L-1)$ opened orders over $F$. We conclude that the Median Assignment can be completed successfully.

COROLLARY 4.1 The overall holding cost incurred by the algorithm is at most $2 \sum_{i=1}^{N} \sum_{t=1}^{T} \sum_{s=1}^{t} \hat{x}_{s t}^{i} H_{s t}^{i}$.

Lemma 4.1 and Corollary 4.1 imply the following theorem.

THEOREM 4.1 The Random-Shift algorithm is a randomized 2-approximation algorithm for the capacitated multi-item lot-sizing problem with hard capacities.

We note that the same analysis holds in the presence of soft capacities, where we think on each batch as a separate potential order. It can be verified that all the arguments in the analysis presented above still go through.

Finally, we describe how to derandomize the algorithm and get deterministic approximation algorithm with the same worst-case performance guarantee. We have already mentioned that once the periods in which orders are placed are determined, the problem is reduced to solving a transportation problem that minimizes the holding costs. The analysis in Section 4.2 implies that for any outcome of the RandomShift procedure the induced transportation problem is feasible, and its optimal solution incurs holding costs that are at most twice the holding costs incurred by the optimal fractional solution $(\hat{x}, \hat{y})$. That is, this happens with probability 1 . It is now sufficient to show how to derandomize Phase I of the algorithm. However, it is readily verified that in the Random-Shift procedure described above, there is only a polynomial number of values of $\alpha$ that yield distinct sets of orders. Specifically, there are $O(T)$ such points. Moreover, these values can be easily enumerated. Thus, the cost of the solution obtained by taking the best (cheapest) among the different solutions is at most the expected cost over all choices. 
THEOREM 4.2 There exists a deterministic 2-approximation algorithm for the capacitated multi-item lotsizing problem with hard capacity constraints.

4.3 On-The-Fly Algorithm In this section, we shall describe an on-the-fly variant of the algorithm described above. The underlying idea is similar to what discussed by Carr et al. [8] in the context of the fixed-charge single-node problem.

This variant does not require solving the LP a-priori with all the flow-cover inequalities defined by the collection of subsets $\mathcal{F}$ in Section 3. Instead, we shall have an iterative procedure that is based on an oracle that, in each iteration, either finds a violated flow-cover inequality or generates a feasible solution with cost that is at most twice the optimal cost. In particular, in each iteration, we attempt to apply the rounding algorithm described in Sections 4.1 and 4.2 using the Median Assignment procedure. If the Median Assignment procedure is terminated successfully we are guaranteed to have an integer solution with cost at most twice the optimal cost. Otherwise, we have identified a violated inequality that can be added to the LP, which is then being resolved. We note that in practice the on-the-fly algorithm can be implemented using the Simplex method. Since in each iteration we add a constraint to the primal LP, the Dual-Simplex method might be very attractive to find the new optimal solution of the LP. However, note that this algorithm is not guaranteed to terminate after polynomial number of iterations.

Alternatively, we can run the Ellipsoid method using the Median Assignment procedure as a separation oracle instead of the separation algorithm described in Section 3. Having an efficient oracle that can separate the respective flow-cover inequalities enables us to run the Ellipsoid method to solve an implicit LP, that contains only a subset of the flow-cover inequalities that define the LP described in Section 3. Moreover, this implicit LP provides a lower bound on the optimal cost, and its optimal fractional solution can be rounded to an integer solution with cost at most twice the optimal cost.

5. Conclusions In this paper, we study the classical capacitated multi-item lot-sizing problem with hard capacities, and propose a novel LP-based 2-approximation algorithm for the problem. The LP relaxation being used for the construction of the algorithm and in the worst-case analysis is based on the well-known flow-cover inequalities.

Beyond the algorithmic results, this paper is the first to establish a theoretical analysis of the strength of flow-cover inequalities in capacitated inventory models. Furthermore, we believe that some of the novel algorithmic ideas proposed in this paper have a promising potential in constructing strong LP relaxations and LP-based approximation algorithms for other inventory models, and for the capacitated facility location problem.

In addition, the on-the-fly variant of the algorithm might be computationally attractive, and can be easily incorporated into existing integer programming solution procedures, such as Branch and Bound and Branch and Cut. It will be very interesting to explore this direction. It will also be very interesting to use the LP proposed in this paper to construct combinatorial algorithms via a primal-dual approach.

We note that other variants of flow-cover inequalities can be used to construct LP relaxations for the model with nonuniform capacities. However, rounding the fractional solution in this setting seems to be more challenging. Finally, we note that in the presence of fixed item ordering cost, the LP relaxation proposed in this paper has an unbounded integrality gap. Constructing strong LP relaxations and approximation algorithms for this model seems to require fundamentally new ideas.

Acknowledgments. Part of this research has been carried out when the first and the second authors were Herman Goldstine Postdoctoral Fellows in the Department of Mathematical Sciences of the IBM T.J. Watson Research Center, whose support is strongly acknowledged.

The research of the first author was partially supported by the IBM Faculty Award and NSF grant DMS-0732175. The reseach of the second author was partially supported by the EU projects ADONET (contract n. MRTN-CT-2003-504438) and ARRIVAL (contract n. FP6-021235-2).

We warmly thank Oktay Günlük for pointing out our attention to reference [5], and David Shmoys for numerous fruitful discussions. We all thank the three anonymous referees for a careful reading and very useful comments. 


\section{References}

[1] K. Aardal. Capacitated facility location: separation algorithms and computational experience. Mathematical Programming, 81:149-175, 1998.

[2] K. Aardal, Y. Pochet, and L. A. Wolsey. Capacitated facility location: valid inequalities and facets. Mathematics of Operations Research, 20:562-582, 1995.

[3] S. Anily and M. Tzur. Shipping multiple-items by capacitated vehicles - an optimal dynamic programming approach. Transportation Science, 39:233-248, 2005.

[4] S. Anily, M. Tzur, and L. A. Wolsey. Multi-item lot-sizing with joint set-up cost. Technical Report 2005/70, CORE, 2005. Working paper.

[5] A. Atamtürk and D. Rajan. On splittable and unsplittable flow capacity network design arc-set polyhedra. Mathematical Programming, 92:315-333, 2002.

[6] G. R. Bitran and H. H. Yanasee. Computational complexity of the capacitated lot-size problem. Management Science, 28:1174-1186, 1982.

[7] T. Carnes and D. B. Shmoys. A primal-dual 2-approximation algorithm the single-demand fixed-charge minimum-cost flow problem. Working paper, 2006.

[8] R. D. Carr, L. K. Fleischer, V. J. Leung, and C. A. Phillips. Strengthening integrality gaps for capacitated network design and covering problems. In Proceedings of the 11th ACM/SIAM Symposium on Discrete Algorithms (SODA), 2000.

[9] G. Even, R. Levi, D. Rawitz, B. Schieber, S. Shahar, and M. Sviridenko. Algorithms for capacitated rectangle stabbing and lot-sizing with joint set-up costs. Working paper, 2006.

[10] A. Federgruen and J. Meissner. Probabilistic analysis of multi-item capacitated lot sizing problems. Working paper, 2004.

[11] A. Federgruen, J. Meissner, and M. Tzur. Progressive interval heuristics for the multi-item capacitated lot sizing problem. Operations Research, 55, 2007.

[12] M. Florian, J. K. Lenstra, and A. H. G. Rinooy Kan. Deterministic production planning: Algorithms and complexity. Management Science, 26:669-679, 1980.

[13] M. Garey and D. S. Johnson. Computers and intractability. A guide to the theory of NP-completeness. W. H. Freeman and Co., San Francisco, California, 1979.

[14] R. Levi, R. O. Roundy, and D. B. Shmoys. Primal-dual algorithms for deterministic inventory problems. Mathematics of Operations Research, 31:267-284, 2006.

[15] R. Levi, R. O. Roundy, D. B. Shmoys, and M. Sviridenko. First constant approximation algorithm for the single-warehouse multi-retailer problem. Technical Report 1408, School of Operations Research and Industrial Engineering, Cornell University, 2004. To appear in Management Science.

[16] T. L. Magnanti, P. Mirchandani, and R. Vachani. The convex hull of two core capacitated network design problems. Mathematical Programming, 60:233-250, 1993.

[17] G. Nemhauser and L. A. Wolsey. Integer Programming and Combinatorial Optimization. Wiley, 1990.

[18] M. W. Padberg, T. J. V. Roy, and L. A. Wolsey. Valid inequalities for fixed charge problems. Operations Research, 33:842-861, 1985.

[19] Y. Pochet and L. A. Wolsey. Lot-sizing with constant batches: formulation and valid inequalities. Mathematics of Operations Research, 18:767-785, 1993.

[20] Y. Pochet and L. A. Wolsey. Production Planning by Mixed Integer Programming. Springer Verlag, 2006.

[21] C. P. M. van Hoesel and A. P. M. Wagelmans. Fully polynomial approximation schemes for single-item capacitated economic lot-sizing problems. Mathematics of Operations Research, 26:339-357, 2001. 